\title{
Hybrid position/force control of a spatial compliant mechanism
}

\author{
E. Shojaei Barjuei
}

\author{
Department of Advanced Robotics, \\ Istituto Italiano di Tecnologia (IIT), 16163 Genoa, ITALY \\ Email: erfan.shojaei@iit.it \\ Phone: +3901071781501; Fax: +3901071781231
}

\begin{abstract}
This paper provides a hybrid position/force control design for a flexible L-shape mechanism in a three dimensional (3D) environment. The analytical mechanism model was developed with a highly accurate dynamic system based on equivalent rigid link system theory and finite element discretisation without the need for any dedicated force/torque sensors. The gravity force and dynamic model nonlinear parameters were taken into account. This paper also presents and describes a real-time approach based on a comparison between joint acceleration and the time evolution of a simplified rigid mechanism model for estimating external forces applied on the tip of the mechanism. The most significant simulation results are presented and discussed; in particular, position controller has a high accuracy in terms of trajectory tracking with max error of 2.6 degree.
\end{abstract}

Keywords: Flexible-link mechanism, dynamic model; hybrid position/force control; trajectory tracking; external force regulation.

\section{INTRODUCTION}

Control of compliant mechanisms is a research area that has aroused remarkable interest due to its vast application in automated manufacturing and robotics systems. The majority of robotic applications found in industry are based on simple position-controlled manipulators that use joint position feedback to close the control loops such as spray painting and pick-and-place tasks. Although the application of position control is well suited for the tasks in which the robot is not constrained by any object in the workspace; a successful and safe interaction between a robot and its environment is required in many automated manufacturing systems, sometimes within a non-explicitly known environment. According to [1], interaction force and motion control in robotics are generally divided in two main approaches; impedance control and hybrid motion/force control. While impedance control [2] stands for adjusting the mechanical impedance of the contact point with a rigid environment, the main concept of hybrid position/force control [3] is based on subjecting the end effector of the manipulator to external constraints which are experienced in the form of interaction forces when the manipulator interacts with the environment. Accordingly, interaction forces should be accommodated rather than resisted [4] so the control approach should handle tracking error properly to improve the contact. Moreover, hybrid position/force control provides better performance respect to impedance control for robotic tasks that need high accuracy and precision [5]. According to [6], the main advantage of hybrid position/force control with respect to other active force controls is that the position and force data are analysed independently to 
benefit from the dedicated control technique for each one. Most control approaches, which lie in interaction force and motion of flexible link manipulators use force/torque sensors mounted at the robot wrist [7]. Some approaches for force estimation by means of the signals obtained from strain measurements (mechanical deformation) for different dynamic systems were presented in $[8,9]$ as well. For making the estimation of the applied external force to a flexible mechanism, approaches based on the disturbance observer were presented in $[10,11]$. Nevertheless, in aforementioned approaches, the use of measurement sensors was still needed and the performance and the robustness of the observer depended on not only to the precision of tuning parameters but also the accuracy of the dynamic model. On the other hand, force recognition with means of the inverse method is very important when direct measurement using force sensors is not possible due to technical difficulties and limitation of installation and dynamic characteristic altering problem [12].

To the best of author's knowledge, an application of hybrid force/position control to a flexible mechanism in a three dimensional (3D) environment under gravity force has not been presented yet. The main goal of this paper is to present a dynamic hybrid position/force control approach for a spatial flexible L-shape mechanism with consideration of gravity force into dynamic modelling formulation of the mechanism in the absence of any kind of force/torque sensors. Consequently, the main objective is to allow the tip of the mechanism to safely contact objects in an uncertain environment. In this work, the Equivalent Rigid Link System (ERLS) approach was used for dynamic modelling of the flexible link mechanism in 3D environment [13].The main advantages of the ERLS approach is that the standard concepts of 3D kinematics can be taken to formulate and solve the ERLS kinematics, e.g. Denavit-Hartenberg convention. The obtained results can then be applied and integrated simply in the equations of the dynamic model of the spatial flexible mechanism. Furthermore, the dynamic model of the L-shape mechanism used in this work as a benchmark mechanism was validated experimentally in [14] and also used in other works by the same author such as $[15,16]$.

In this paper, the synthesis of a hybrid/position force control of a flexible mechanism in the 3D environment with gravity is proposed. At first, the dynamic modelling of flexible mechanisms according to the ERLS approach and the reference mechanism used in this work are described. Then the external force estimation technique and hybrid position/force control approach applied in this work are explained as well. Finally, the most significant results are presented and discussed and the conclusion is drawn accordingly.

\section{METHODS AND MATERIALS}

\section{Dynamic Model}

The approach used for the modelling of deformable mechanisms with large displacements and small elastic deformation is based on Equivalent Rigid Link System (ERLS) concept; such model has been introduced and validated for a flexible mechanism in 3D environment by Vidoni in [13]. In this section, this approach is briefly recalled. 


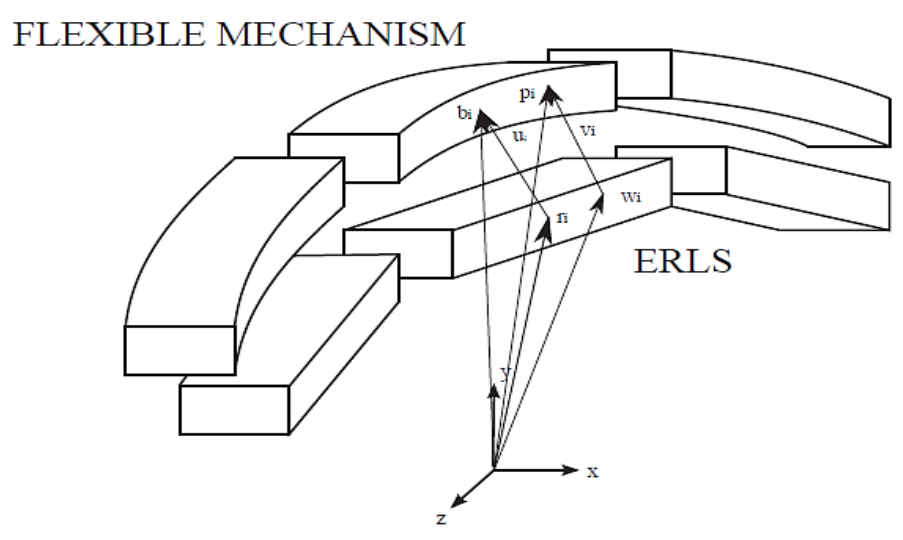

Figure 1. Kinematic definition of the ERLS.

As shown in Figure 1, in order to obtain a model with a finite number of degrees of freedom, each link of the mechanism is divided into finite elements. Furthermore, the whole motion of each compliant link is divided into the large rigid-body motion of an equivalent rigid-link system (ERLS) and the small elastic deflection of the link based on the ERLS itself. Specifically, the following definitions are defined:

- $\quad \boldsymbol{r}_{i}$ and $\boldsymbol{u}_{i}$ represent the vectors of the positions of the nodes in the $i-t h$ element of the ERLS and their elastic displacements respectively; the sum of these vectors brings up the global motion of the nodes of the, $i-t h$ element.

- $\boldsymbol{p}_{i}$ represents the position vector of a point of the finite $i-t h$ element.

- $\quad \boldsymbol{q}$ represents the vector of the ERLS generalised coordinates.

All of these vectors are defined in a global fixed reference frame; a local reference frame following the motion of the ERLS for each element is also used in order to simplify the application of the finite element method.

The dynamic mathematical equations are obtained by applying the concept of virtual work and computing the terms related to inertial, elastic, and external generalised forces:

$$
\sum_{i} \int_{v_{i}} \delta \boldsymbol{p}_{i}^{T} \ddot{\boldsymbol{p}}_{i} \rho_{i} d v+\sum_{i} \int_{v_{i}} \delta \varepsilon_{i}^{T} \boldsymbol{D}_{i} \varepsilon_{i} d v=\sum_{i} \int_{v_{i}} \delta \boldsymbol{p}_{i}^{T} \mathbf{g} \rho_{i} d v+\left(\delta \boldsymbol{u}^{T}+\delta \boldsymbol{r}^{T}\right) \boldsymbol{f}
$$

In which, $\rho_{i}, \boldsymbol{D}_{i}$ and $\epsilon_{i}$ are the mass density, the stress-strain matrix, and the strain vector for the $i$ - $t h$ element, respectively, $\boldsymbol{g}$ is the gravity acceleration vector and $\boldsymbol{f}$ is the vector of the concentrated external forces and torques; $\delta \boldsymbol{u}$ and $\delta \boldsymbol{r}$ are the virtual displacement related to all nodes of the model. The following formulations are used for the virtual displacement and real acceleration of a generic point belonging to the mechanism:

$$
\begin{gathered}
\delta \boldsymbol{p}_{i}=\boldsymbol{R}_{i} \boldsymbol{N}_{i} \boldsymbol{T}_{i} \delta \boldsymbol{r}_{i}+\boldsymbol{R}_{i} \boldsymbol{N}_{i} \boldsymbol{T}_{i} \delta \boldsymbol{u}_{i} \\
\ddot{\boldsymbol{p}}_{i}=\boldsymbol{R}_{i} \boldsymbol{N}_{i} \boldsymbol{T}_{i} \ddot{\boldsymbol{r}}_{i}+\boldsymbol{R}_{i} \boldsymbol{N}_{i} \boldsymbol{T}_{i} \ddot{\boldsymbol{u}}_{i}+2\left(\dot{\boldsymbol{R}}_{i} \boldsymbol{N}_{i} \boldsymbol{T}_{i}+\boldsymbol{R}_{i} \boldsymbol{N}_{i} \dot{\boldsymbol{T}}_{i}\right) \dot{\boldsymbol{u}}_{i}
\end{gathered}
$$

where $\boldsymbol{T}_{i}$ is the transformation matrix from the global to the local reference frame for the $i$ - th element and $R_{i}$ is the local-to-global rotation matrix; $\boldsymbol{N}_{i}$ is the shape function matrix, which is defined in the local frame. The strain displacement matrix $\boldsymbol{B}_{i}$ can be introduced on the basis of real and virtual strains as follows: 


$$
\begin{gathered}
\varepsilon_{i}=\boldsymbol{B}_{i} \boldsymbol{T}_{i} \boldsymbol{u}_{i} \\
\delta \epsilon_{i}=\boldsymbol{B}_{i} \delta \boldsymbol{T}_{i} \boldsymbol{u}_{i}+\boldsymbol{B}_{i} \boldsymbol{T}_{i} \delta \boldsymbol{u}_{i}
\end{gathered}
$$

Since the nodal elastic virtual displacements $(\delta \boldsymbol{u})$ and the nodal virtual displacement of the ERLS $(\delta \boldsymbol{r})$ are independent from each other, the dynamic model of a generic flexible-link mechanism can be written as:

$$
\left[\begin{array}{cc}
\boldsymbol{M} & \boldsymbol{M S} \\
\boldsymbol{S}^{T} \boldsymbol{M} & \boldsymbol{S}^{T} \boldsymbol{M S}
\end{array}\right]\left[\begin{array}{l}
\ddot{\boldsymbol{u}} \\
\ddot{\boldsymbol{q}}
\end{array}\right]=\left[\begin{array}{ccc}
-2 \boldsymbol{M}_{G}-\alpha \boldsymbol{M}-\beta \boldsymbol{K} & -\boldsymbol{M} \dot{\boldsymbol{S}} & -\boldsymbol{K} \\
\boldsymbol{S}^{T}\left(-2 \boldsymbol{M}_{G}-\alpha \boldsymbol{M}\right) & -\boldsymbol{S}^{T} \boldsymbol{M} \dot{\boldsymbol{S}} & 0
\end{array}\right]\left[\begin{array}{c}
\dot{\boldsymbol{u}} \\
\dot{\boldsymbol{q}} \\
\boldsymbol{u}
\end{array}\right]+\left[\begin{array}{cc}
\boldsymbol{M} & I \\
\boldsymbol{S}^{T} \boldsymbol{M} & \boldsymbol{S}^{T}
\end{array}\right]\left[\begin{array}{l}
\boldsymbol{g} \\
\boldsymbol{f}
\end{array}\right](6)
$$

where matrix $\boldsymbol{M}_{G}$ allows keeping into account the Coriolis contributions, $\boldsymbol{M}$ is the mass matrix of the whole system, $\boldsymbol{K}$ is the matrix obtained when assembling the stiffness matrices of all elements and $\boldsymbol{S}$ is the sensitivity matrix for all the nodes. A Rayleigh model of damping has been considered and inserted in the model through the $\alpha$ and $\beta$ constants.

By solving the system Equation (6), accelerations can be computed and by means of an appropriate integration scheme, velocities and displacements can be obtained [13].

\section{Reference Mechanism}

The mechanism chosen as the basis of the simulations is a compliant L-shape mechanism made by two steel rods connected by a rigid aluminium joint (Figure 2). The rotational motion of the first link can be imposed through a torque-controlled actuator. The whole mechanism can swing in 3D environment and is described by three finite-elements; that is, the link connected to electrical motor is modelled with two finite-elements while the other link is described by a single finite element. The experimental validation of the dynamic model used in this work was performed in [14].

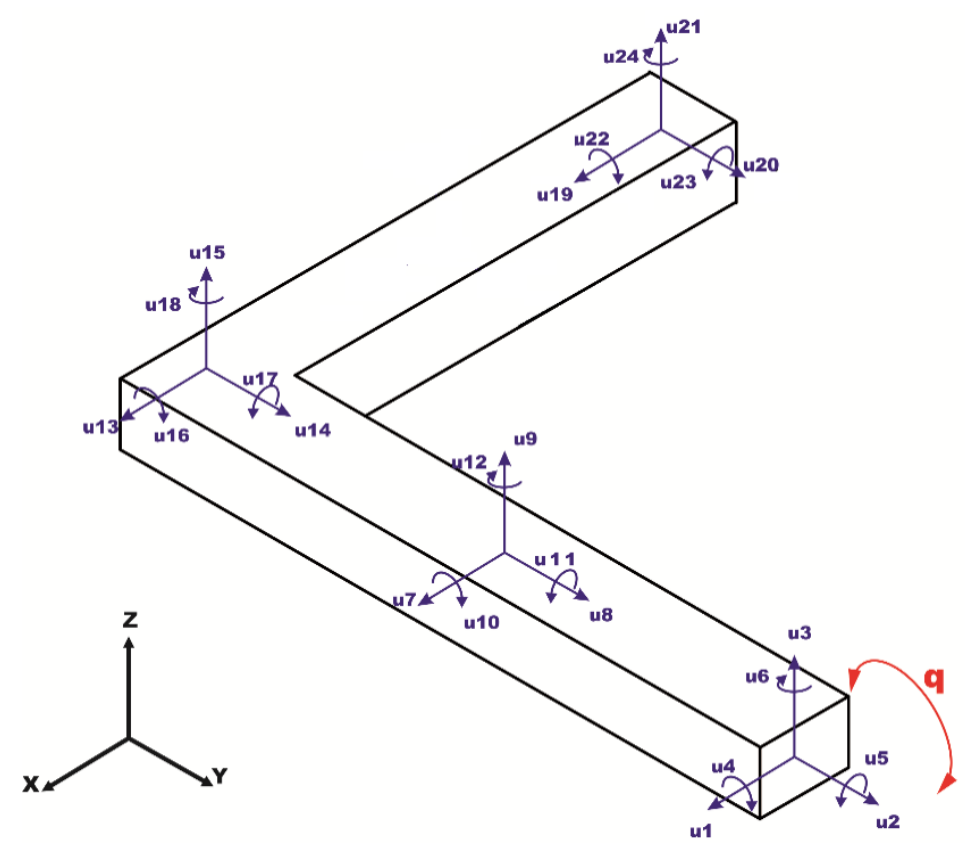

Figure 2. Elastic displacement in the flexible L-shape mechanism. 
The mechanical parameters of the flexible L-shape mechanism components are reported in Table 1. In this work, a software program for simulating the dynamics behaviour of a flexible-link has been developed based on simulator presented in [17] and also according to the dynamic model described in the previous section.

Table 1. Kinematics and dynamics characteristic of the reference mechanism.

\begin{tabular}{lcc}
\hline \multicolumn{1}{c}{ Description } & Symbol & Value \\
\hline Young's modulus & $E$ & $2 \times 10^{11}[\mathrm{~Pa}]$ \\
Flexure inertia moment & $J$ & $11.10^{2} \times 10^{-10}\left[\mathrm{~m}^{4}\right]$ \\
Poisson's coefficient & $v$ & 0.33 \\
Beam width & $a$ & $30 \times 10^{-3}[\mathrm{~m}]$ \\
Beam thickness & $b$ & $10 \times 10^{-3}[\mathrm{~m}]$ \\
Density & $\varphi$ & $7850\left[\mathrm{Kg} / \mathrm{m}^{3}\right]$ \\
First link length & $L_{1}$ & $0.5[\mathrm{~m}]$ \\
Second link length & $L_{2}$ & $0.5[\mathrm{~m}]$ \\
Rayleigh damping constant & $\alpha$ & $7 \times 10^{-4}\left[\mathrm{~s}^{-1}\right]$ \\
& $\beta$ & $2.13 \times 10^{-7}[\mathrm{~s}]$ \\
\hline
\end{tabular}

\section{External Force Estimation}

In this section, an approach to the external force estimation based on the comparison between the motion of the flexible mechanism and its rigid counterpart is presented. Figure 3 shows a schematic view of the proposed approach. As it can be seen from Figure 3, while the external force and torque produced by the electrical motor are applied to the flexible mechanism, only the torque produced by the electrical motor is applied to the rigid mechanism. Furthermore, joint accelerations of both of the mechanisms are measured and compared to each other.

By assuming that the external force is applied on the tip of the mechanism along the tangential direction (Figure 2), the dynamic model stated in Equation (6) can be rewritten as:

so that

$$
\boldsymbol{M}_{E R L S}(q, \dot{q})\left[\begin{array}{c}
\ddot{\boldsymbol{u}} \\
\ddot{q}_{f l e x}
\end{array}\right]=\boldsymbol{A}(\boldsymbol{u}, \dot{\boldsymbol{u}}, q, \dot{q})\left[\begin{array}{c}
\dot{\boldsymbol{u}} \\
\dot{q} \\
\boldsymbol{u}
\end{array}\right]+\boldsymbol{B}(q, \dot{q})\left[\begin{array}{l}
\boldsymbol{g}_{\boldsymbol{f}} \\
\boldsymbol{f}
\end{array}\right]
$$

$$
\left[\begin{array}{c}
\ddot{\boldsymbol{u}} \\
\ddot{q}_{f l e x}
\end{array}\right]=\boldsymbol{M}_{E R L S}^{-1}(q, \dot{q}) \boldsymbol{A}(\boldsymbol{u}, \dot{\boldsymbol{u}}, q, \dot{q})\left[\begin{array}{c}
\dot{\boldsymbol{u}} \\
\dot{q} \\
\boldsymbol{u}
\end{array}\right]+\boldsymbol{M}_{E R L S}^{-1}(q, \dot{q}) \boldsymbol{B}(q, \dot{q})\left[\begin{array}{l}
\boldsymbol{g} \\
\boldsymbol{f}
\end{array}\right]
$$

Equation (8) is the explicit form of the flexible mechanism dynamic model, which can be used to evaluate the joint acceleration $\left(\ddot{\boldsymbol{q}}_{\text {flex }}\right)$. The vector of external forces $(\boldsymbol{f})$ in Equation (7) includes both the torque $\left(\tau_{m}\right)$ provided by the actuator and the external force $\left(f_{\text {ext }}\right)$ applied on the tip of the mechanism.

According to Equation (8), joint acceleration $\ddot{q}_{\text {flex }}$ can be expressed as:

$$
\ddot{q}_{\text {flex }}(t)=f\left(q, \dot{q}, u, \dot{u}, \tau_{m}, f_{\text {ext }}\right)
$$

By neglecting the effects of flexibility in Eq. (9), $\ddot{q}_{\text {flex }}$ can be written as: 


$$
\ddot{q}_{\text {flex }}(t) \simeq \frac{1}{J} \tau_{m}(t)+\frac{1}{J} L_{1} f_{\text {ext }}(t)
$$

where $L_{1}$ is the length of the first link and $J$ represents the moment of inertia of the mechanism. In the absence of external forces, the angular acceleration can be written simply as:

$$
\ddot{q}_{\text {rigid }}^{*}=\frac{1}{J} \tau_{m}
$$

Finally, an estimation of the external force $\hat{f}_{\text {ext }}$ acting on the tip of the mechanism along the tangential direction can be achieved by comparing Equation (10) and (11) as follows:

$$
\hat{f}_{\text {ext }}=\left(\ddot{q}_{\text {flex }}-\ddot{q}_{\text {rigid }}^{*}\right) \frac{J}{L_{1}}=\left(\ddot{q}_{\text {flex }}-\frac{1}{J} \tau_{m}\right) \frac{J}{L_{1}}
$$

Equation (12) provides the means of estimating the amplitude of the external force from the measurement of the angular acceleration of the mechanism and the measurement of the torque produced by the actuator. When neglected, link flexibility can lead to large estimation errors [18].

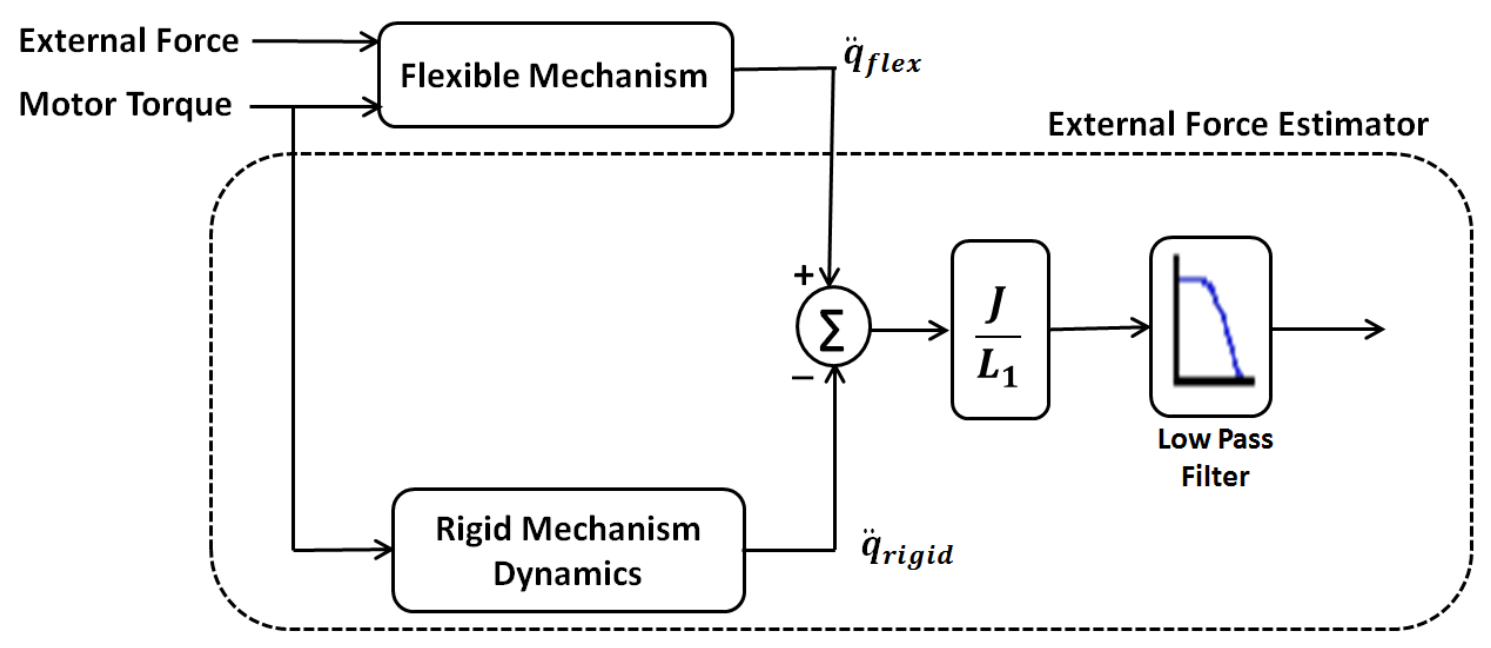

Figure 3. Schematic view of the external force estimation approach.

\section{Hybrid Position/Force Control}

In this section, a short description of the hybrid position/force control for the L-shape flexible mechanism is given. Hybrid position/force control combines position and force data into the control loop for moving the mechanism in a non-deterministic environment. The block diagram of hybrid position/force control approach used in this work is shown in Figure 4; accordingly, the main idea is to separate the position control from force control and combine the achieved data. While the angular position of the mechanism $(q)$ is gathered from an encoder and compared to the reference position signal in order to measure the position error, the joint acceleration of the mechanism $\left(\ddot{q}_{\text {flex }}\right)$ is used for estimating the external force applied to the tip of the mechanism according to the technique explained in the previous section. The estimated external force is used in the following control law: 


$$
\Delta(t)=\int_{0}^{\mathrm{t}} \hat{f}_{\text {ext }}(\mathrm{t}) \Theta d t
$$

where $f_{\text {ext }}$ is the estimation of the external applied force on the tip of the mechanism, $\Theta$ is a positive constant with value of $5 \frac{\mathrm{deg}}{N . t}$. In fact, $\Delta(t)$ is used to change the reference position based on the direction and magnitude of the external force applied to the tip of the flexible mechanism. The value of $\Theta$ can be chosen arbitrarily based on the desired performance.

If $\Delta(t)$ is equal to zero, the control loop in Figure 4 acts as tracking joint position control based on PID controller with $K_{p}=5, K_{I}=4, K_{D}=4$. If $\Delta(t)$ is either positive or negative, the joint reference position is altered in order to regulate the contact force to zero. For example, by applying a $1 \mathrm{~N}$ force for $2 \mathrm{sec}$ to the tip of the mechanism along the tangential direction (Figure 2), the mechanism should be moved by $10 \mathrm{deg}$ from its initial configuration in the same direction of the force.

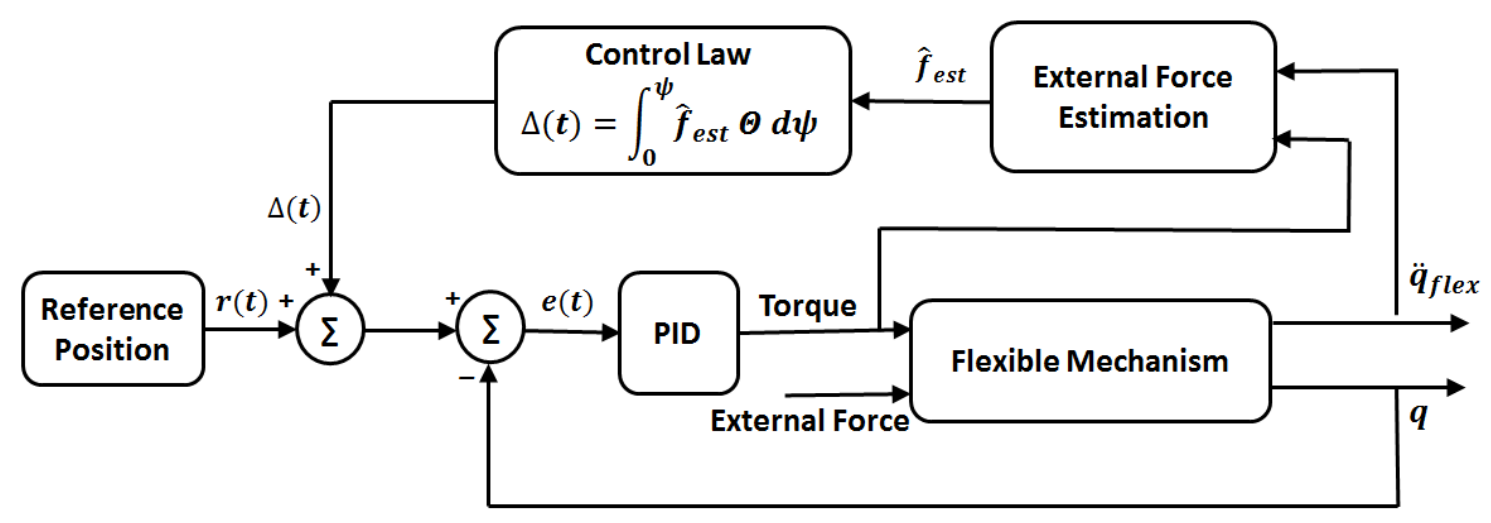

Figure 4. Hybrid position/force control scheme for spatial L-shape flexible mechanism.

\section{RESULTS AND DISCUSSION}

\section{External Force Estimation Technique}

The design of the external force estimation implemented in this work is based on the comparison between the motion of the flexible mechanism and its rigid counterpart. Figures 5 and 6 show the comparison between non-filtered and estimated force with actual external force applied on the tip of the spatial L-shape flexible mechanism respectively. Figure 5 illustrates the comparison between the actual and estimated force applied on the tip of the mechanism along the tangential direction. It can be clearly seen that a pronounced error is introduced by the neglected flexibility of the estimation model. Therefore, a second low order pass filter with cut-off frequency of $20 \mathrm{rad} / \mathrm{s}$ was used to improve the accuracy of the external force estimation. Figure 6 shows a comparison of the estimated and actual force applied on the tip of mechanism when using a low pass filter. It can be inferred that the estimation is quite satisfactory and efficient; however, the lagging difference (around $0.2 \mathrm{sec}$ ) between two curves is due to the low-pass filtering action included in the force estimator. 


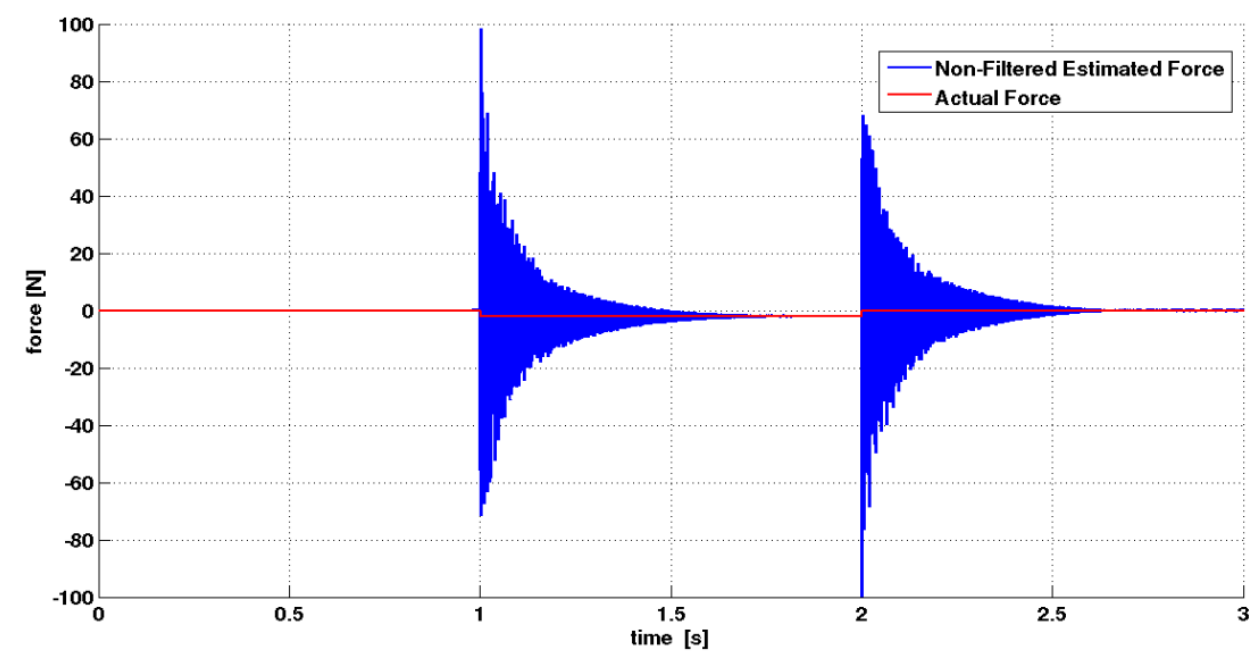

Figure 5. Comparison between non-filtered estimated and actual external force applied on the tip of mechanism.

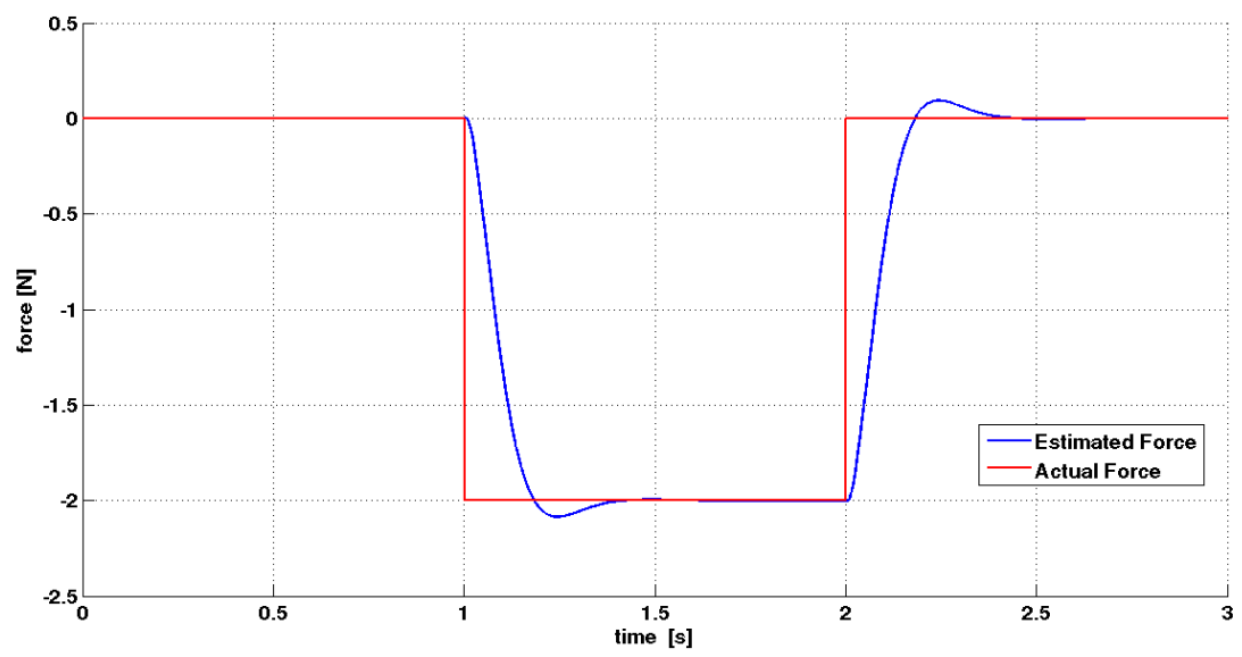

Figure 6. Comparison between estimated and actual external force applied on the tip of mechanism.

\section{Hybrid Position/Force Control Approach}

In this section, the results of the application of the hybrid position/force control approach to the L-shape flexible mechanism in 3D environment are presented. Hence, a force signal with pulse width of $1 \mathrm{sec}$ and amplitude of $-2 N$ shown in Figures 4 and 5 is applied to the tip of the mechanism; while, the initial angular position reference is set to $90 \mathrm{deg}$. As it can be seen from Figure 7, the mechanism starts to move in the same direction of the external force as soon as the latter is applied. The angular displacement of the mechanism was equal to $10 \mathrm{deg}$; in other words, the mechanism moved from its initial configuration (90 deg) to the configuration of $80 \mathrm{deg}$; such displacement is determined by the control law in Equation (12). As can be seen from Figure 7, the control-loop has a fast response with a highly accurate tracking behaviour. From Figure 8, it can be inferred that the position controller has a good accuracy in terms of trajectory tracking and the max error between the actual joint position while the reference position was very small, which was near to $2.6 \mathrm{deg}$ that was $0.26 \%$ of $10 \mathrm{deg}$. Furthermore, according to Figure 9, the overall 
torque produced by electric motor was always within the $\pm 2.5 \mathrm{Nm}$ range which was much lower than the desired limit. It must be noticed that torque by the controller was kept below the limit values for the torques of the electrical motor actuating the real mechanism, i.e. $20 \mathrm{Nm}$.

It can also be inferred that the performance of the hybrid position/force controller was found to be particularly affected by the PID controller gains, features of the input signal, type of applied filter, and initial conditions. Additionally, using a proper low-pass filter greatly improved the performance of the system response that can be brought to follow the reference signal more closely.

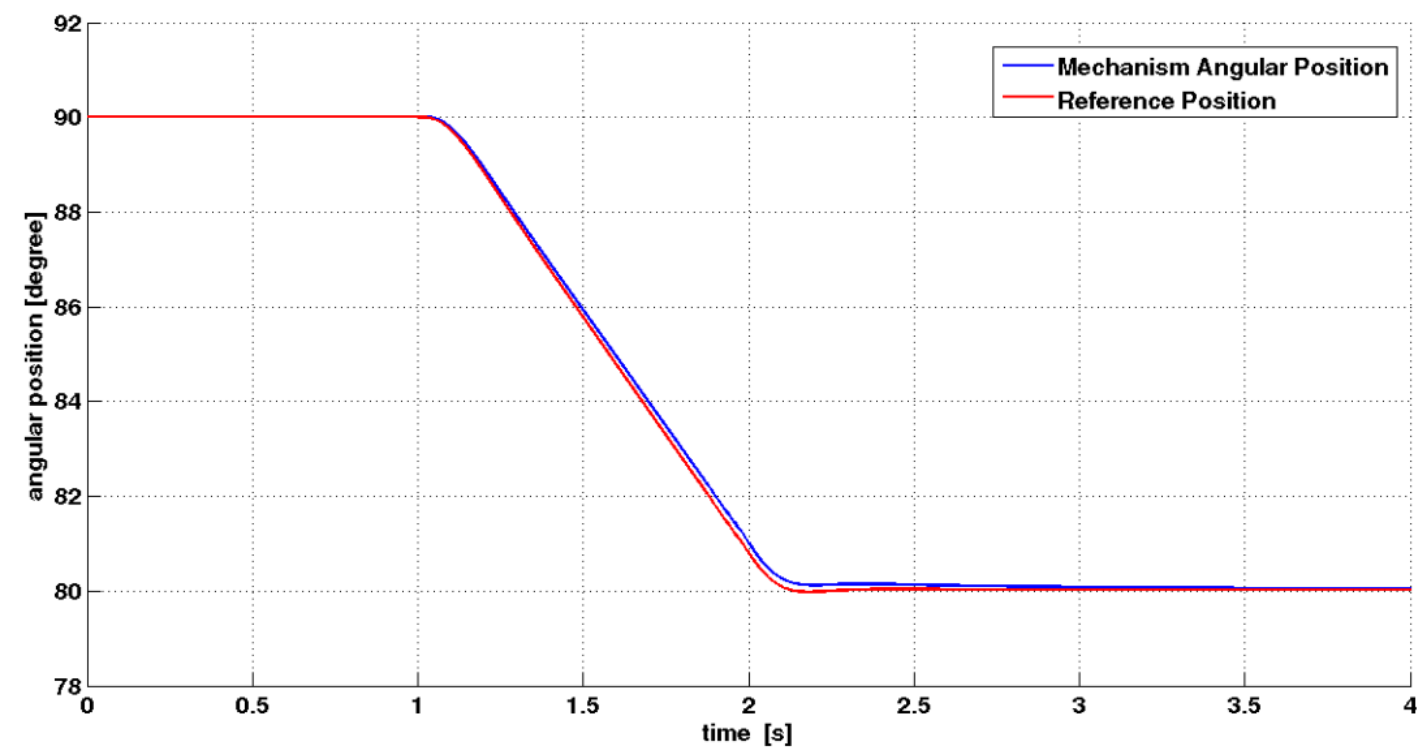

Figure 7. Response of the flexible mechanism to applied external force in terms of angular position.

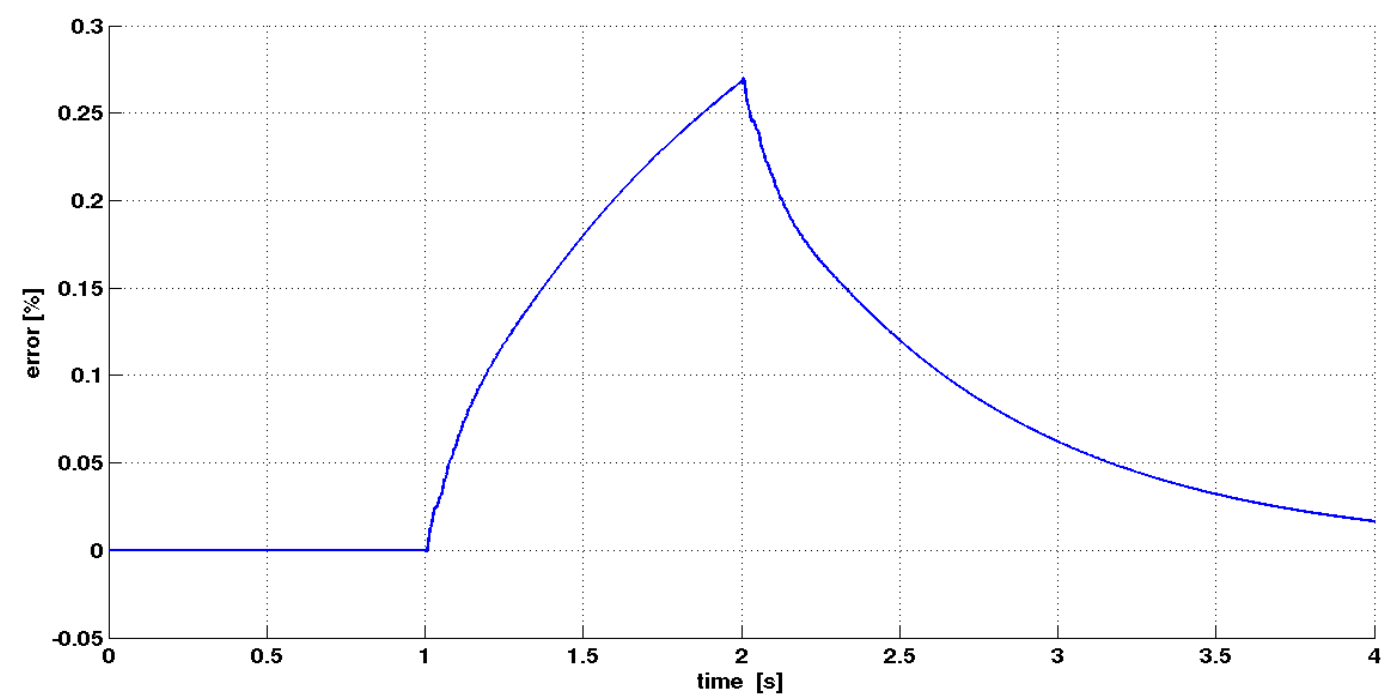

Figure 8. Percentage error on the difference between mechanism angular position and reference position. 


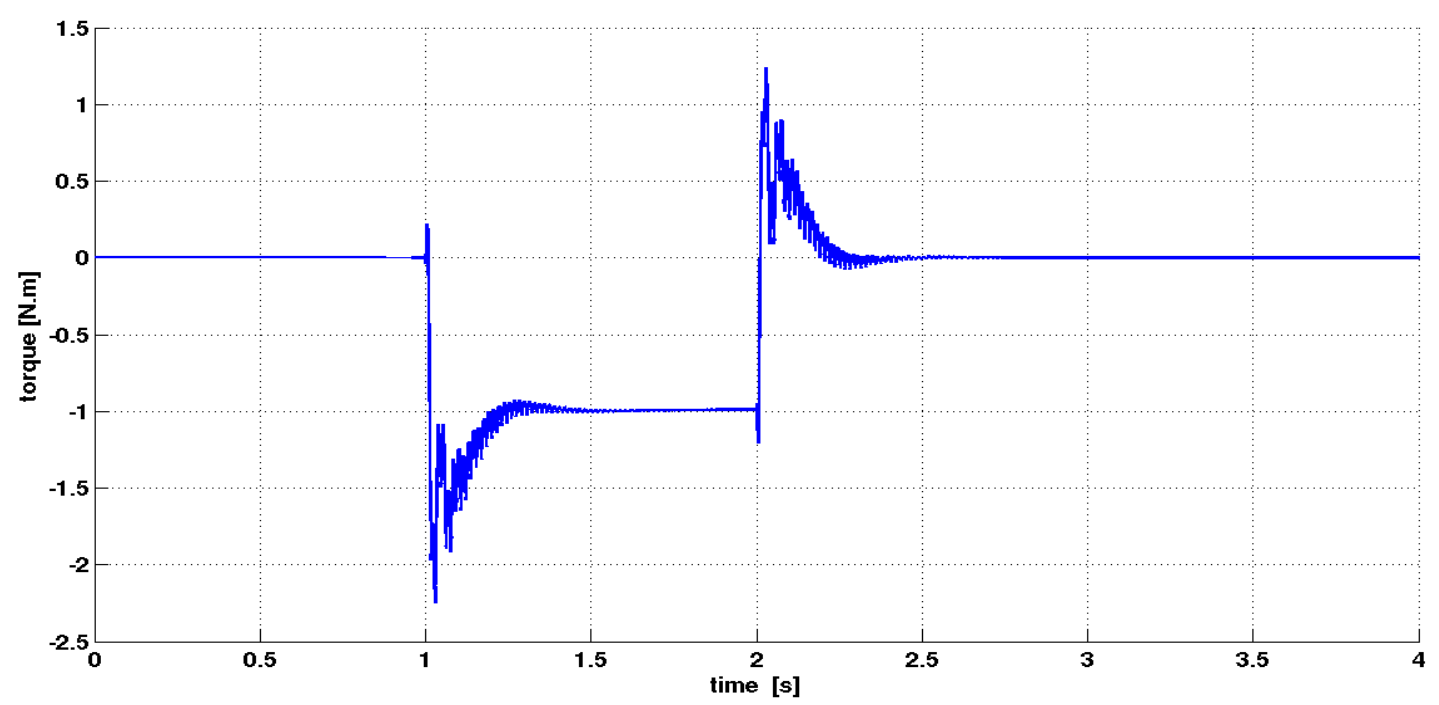

Figure 9. Motor torque.

\section{CONCLUSIONS}

In this paper, a dynamic hybrid position/force control for a flexible L-shape mechanism in 3D environment was synthesised and tested. The mechanism was modelled by means of a highly accurate Finite Element model based on the equivalent rigid link system (ERLS) theory and accounting for geometric nonlinearities and gravity force. In order to implement the hybrid position/force control, an external force estimation approach was presented. The estimation technique was based on the comparison between the actual joint acceleration and the joint acceleration computed through a simplified rigid model. The control scheme was composed by two loops; one performed the angular position tracking, while the other was responsible for the indirect regulation of the interaction forces between the tip of the mechanism and the environment. Hence, the reference position fed to the position controller can be changed according to a control law, which depended on the direction and the amplitude of the external force. The controller demonstrated good trajectory tracking performance and an accurate external force regulation.

\section{ACKNOWLEDGEMENTS}

The author would like to thank the anonymous reviewers for their valuable and constructive suggestions, and their efforts provided in helping to improve the quality of the paper.

\section{REFERENCES}

[[1] Bajo A, Simaan N. Hybrid motion/force control of multi-backbone continuum robots. The International Journal of Robotics Research. 2016;35:422-34.

[2] Hogan N. Impedance Control: An Approach to Manipulation: Part I-Theory. Journal of Dynamic Systems, Measurement, and Control. 1985;107:1-7.

[3] Raibert MH, Craig JJ. Hybrid Position/Force Control of Manipulators. Journal of Dynamic Systems, Measurement, and Control. 1981;103:126-33. 
[4] Jiang T, Liu J, He W. Boundary control for a flexible manipulator based on infinite dimensional disturbance observer. Journal of Sound and Vibration. 2015;348:114.

[5] Yoshikawa T. Force control of robot manipulators. IEEE International Conference on Robotics and Automation Symposia; 2000. p. 220-6.

[6] W. Robinson D. Design and Analysis of Series Elasticity in Closed-loop Actuator Force Control; 2010.

[7] Siciliano B, Villani L. Robot force control: Springer Science \& Business Media; 2012.

[8] Rahman A, Ghaffar A, Yee KS, Ismail Z, Tong CW, Noroozi S. Impact force identification by using modal transformation method for automobile test rig. Trans Tech Publ; 2014. p. 102-6.

[9] Sani MSM, Rahman MM, Noor MM, Kadirgama K, Izham MHN. Identification of Dynamics Modal Parameter for Car Chassis. IOP Conference Series: Materials Science and Engineering. 2011;17:012038.

[10] Atashzar SF, Talebi HA, Towhidkhah F, Shahbazi M. Tracking control of flexible-link manipulators based on environmental force disturbance observer. 49th IEEE Conference on Decision and Control; 2010. p. 3584-9.

[11] Rashidinejad A, Nikravesh SKY, Talebi HA. Nonlinear disturbance observer for flexible-link manipulators based on flexural rate estimation. The 3rd International Conference on Control, Instrumentation, and Automation; 2013. p. 269-73.

[12] Rahman AGA, Yee KS, Ismail Z, Kuan K, Chao O, Chong W, et al. Impact force identification using the modal transformation method in collocated and noncollocated cases. Journal of Mechanical Engineering and Sciences. 2014;6:96874.

[13] Vidoni R, Gasparetto A, Giovagnoni M. Design and implementation of an ERLSbased 3-D dynamic formulation for flexible-link robots. Robotics and ComputerIntegrated Manufacturing. 2013;29:273-82.

[14] Boscariol P, Gasparetto A, Giovagnoni M, Moosavi AK, Vidoni R. On the modeling of flexible-link robots: First experimental validation of an ERLS-FEM dynamic model. IEEE International Conference on Mechatronics; 2013. p. 298302.

[15] Shojaei Barjuei E, Gasparetto A. Predictive Control of Spatial Flexible Mechanisms International Journal of Mechanics and Control. 2015;16:85-96.

[16] Barjuei ES, Boscariol P, Vidoni R, Gasparetto A. Robust Control of ThreeDimensional Compliant Mechanisms. Journal of Dynamic Systems, Measurement, and Control. 2016;138:101009--14.

[17] Boscariol P, Gasparetto A, Giovagnoni M, Moosavi AK, Vidoni R. Design and Implementation of a Simulator for 3D Flexible-Link Serial Robots. 2012:155-64.

[18] Eppinger SD, Seering WP. Three dynamic problems in robot force control. Proceedings, 1989 International Conference on Robotics and Automation; 1989. p. 392-7. 